\title{
Statistical Derivation of the Fundamental Scalar Field
}

\author{
B. I. Lev ${ }^{1,2}$ \\ ${ }^{1}$ Bogolyubov Institute for Theoretical Physics of the NAS of Ukraine, Kyiv, Ukraine \\ ${ }^{2}$ Department of Physics, Chungnam National University, Daejeon, Korea \\ Email: bohdan.lev@gmail.com
}

How to cite this paper: Lev, B.I. (2018) Statistical Derivation of the Fundamental Scalar Field. Journal of Modern Physics, 9, 2223-2232.

https://doi.org/10.4236/jmp.2018.912140

Received: September 14, 2018

Accepted: October 15, 2018

Published: October 18, 2018

Copyright (c) 2018 by author and Scientific Research Publishing Inc. This work is licensed under the Creative Commons Attribution International License (CC BY 4.0).

http://creativecommons.org/licenses/by/4.0/

\begin{abstract}
Based on a nonequilibrium statistical operator, it has been shown that the fundamental scalar field provides a natural representation of the repulsive interaction that produces scattering in the system and thus motivates law of entropy increasing.
\end{abstract}

\section{Keywords}

Statistical Physics, Fundamental Scalar

The standard methods of the equilibrium statistical mechanics cannot be applied to the study of systems with long-range interactions because relevant thermodynamic ensembles can be nonequivalent inasmuch as equilibrium states correspond only to local entropy maxima [1] [2], in particular, since energy is not-additive, and cannot use the canonical ensemble to study the system with long-range interaction. Two types of approaches (statistical and thermodynamic) have been developed to determination the equilibrium states of such interacting system and describe possible phase transition. It is generally believed that mean field theory is exact for systems. Since in mean field theory any thermodynamically function depends on the thermodynamically variables only through the dimensionless combinations and the system is thermodynamically stable but the thermodynamically limit does exist [3].

The purpose of this paper is to develop a new approach [4] [5] to the statistical description of a system of interacting particles with regard for spatial inhomogeneity of the particle or the field distribution. In order to describe such structures, it is necessary to work out a method that would enable us to select the states with thermodynamically stable particle distributions [6]. The main idea is 
to give a detailed treatment of an interacting system in terms of the principles of nonequilibrium statistical mechanics [7]. The representation of the partition function in terms of the functional integral over external fields makes it possible to employ the methods of the quantum field theory [8]-[15]. The extension to the complex plane provides a possibility to apply the saddle-point method to find the dominant contributions to the partition function and to obtain all the thermodynamical functions of the system. It allows selecting the system states associated both with homogeneous and inhomogeneous particle distributions.

This method is based on the Hubbard-Stratonovich representation of the partition function [16]. We employ the saddle-point approximation taking into account the conservation laws for the number of particles and energy and thus obtain a nonlinear equation. The latter provides a statistical description in terms of mathematical physics and may be treated as a natural definition of the field variables. In this way we can answer the question of how the fundamental scalar field appears in the field theory with the necessary condition, to consider the nature of this field and the spatial features of its behavior. The answer to this question should follow from the statistical nature of the interaction and the thermodynamic law of entropy increase.

The phenomenological thermodynamics is based on the conservation laws for the average values of physical parameters, i.e., the number of particles, energy, and momentum. The statistical thermodynamics of nonequilibrium systems is also based on the conservation laws, but for the dynamical variables rather than their average values. It represents local conservation laws for the dynamical variables. In order to determine the thermodynamical functions of a nonequilibrium system, a representation of the relevant statistical ensembles is needed with allowance for the nonequilibrium states of the system. The concept of Gibbs ensemble can provide a description of nonequilibrium stationary states. In this case we can determine a nonequilibrium ensemble as a totality of systems contained under similar stationary external conditions. To determine a local equilibrium ensemble exactly, we have to find the distribution function or the statistical operator of the system [7]. We can assume that nonequilibrium states of the system can be written in terms of the spatial distribution energy $H(\mathbf{r})$ and the microscopic particle density $n(\mathbf{r})=\sum_{i} \delta\left(\mathbf{r}-\mathbf{r}_{i}\right)$. Then the statistical operator [7] of the local equilibrium distribution is given by:

$$
Q_{l}=\int D \Gamma \exp \left\{-\int(\beta(\mathbf{r}) H(\mathbf{r})-\eta(\mathbf{r}) n(\mathbf{r})) \mathrm{d} \mathbf{r}\right\}
$$

The integration in this formula should be carried out over the whole phase space of the system. It should be noted that in the case of local equilibrium distributions the Lagrange multipliers $\beta(\mathbf{r})$ and $\eta(\mathbf{r})$ are functions of spatial points. The local equilibrium distributions may be introduced provided the relaxation time of the whole system is much longer than the relaxation time in any local macroscopic area contained in this system. The thermodynamic relation for interacting systems can be obtained under this condition. The variation of the statistical operator with respect to the Lagrange multipliers yields the 
required thermodynamic relations as given by [7] $-\frac{\delta \ln Q_{l}}{\delta \beta(\mathbf{r})}=\langle H(\mathbf{r})\rangle_{l}$ and $\frac{\delta \ln Q_{l}}{\delta \eta(\mathbf{r})}=\langle n(\mathbf{r})\rangle_{l}$. These relations provide a natural general extension of the well-known relation for equilibrium systems to the case of non-equilibrium systems.

In the general case, the Hamiltonian of a system of interacting particles can be written as

$$
H=\sum_{i} \frac{p_{i}^{2}}{2 m_{i}}+\frac{1}{2} \sum_{i, j} U\left(\mathbf{r}_{i} \mathbf{r}_{j}\right)
$$

where $p_{i}$ and $m_{i}$ is impulse and mass every particle and $U\left(\mathbf{r}_{i} \mathbf{r}_{j}\right)$ determines the energy of the repulsive interaction. The energy density can present in the form:

$$
H(\mathbf{r})=\frac{p^{2}(\mathbf{r})}{2 m(\mathbf{r})} n(\mathbf{r})+\frac{1}{2} \int U\left(\mathbf{r}, \mathbf{r}^{\prime}\right) n(\mathbf{r}) n\left(\mathbf{r}^{\prime}\right) \mathrm{d} \mathbf{r}^{\prime}
$$

The nonequilibrium statistical operator of an interacting system is given by

$$
\begin{aligned}
Q_{l}= & \int D \Gamma \exp \left\{-\int\left(\beta(\mathbf{r}) \frac{p^{2}(\mathbf{r})}{2 m(\mathbf{r})}-\eta(\mathbf{r})\right) n(\mathbf{r}) \mathrm{d} \mathbf{r}\right. \\
& \left.-\frac{1}{2} \int \beta(\mathbf{r}) U\left(\mathbf{r}, \mathbf{r}^{\prime}\right) n(\mathbf{r}) n\left(\mathbf{r}^{\prime}\right) \mathrm{d} \mathbf{r} \mathbf{d} \mathbf{r}^{\prime}\right\}
\end{aligned}
$$

where $D \Gamma=\frac{1}{(2 \pi \hbar)^{3}} \prod_{i} \mathrm{~d} r_{i} \mathrm{~d} p_{i}$ for the integration over the phase space.

In order to perform formal integration in the second term of this presentation, we introduce additional field variables within the context of the theory of Gaussian integrals [8] [16] i.e.,

$$
\begin{aligned}
& \exp \left\{-\frac{v^{2}}{2} \int \beta(\mathbf{r}) \omega\left(\mathbf{r}, \mathbf{r}^{\prime}\right) n(\mathbf{r}) n\left(\mathbf{r}^{\prime}\right) \mathrm{d} \mathbf{r} \mathrm{d} \mathbf{r}^{\prime}\right\} \\
& =\int D \psi \exp \left\{-\frac{v^{2}}{2} \int \beta(\mathbf{r}) \omega^{-1}\left(\mathbf{r}, \mathbf{r}^{\prime}\right) \psi(\mathbf{r}) \psi\left(\mathbf{r}^{\prime}\right) \mathrm{d} \mathbf{r d} \mathbf{r}^{\prime}-v \int \sqrt{\beta(\mathbf{r})} \psi(\mathbf{r}) n(\mathbf{r}) \mathrm{d} \mathbf{r}\right\}
\end{aligned}
$$

where $D \psi=\frac{\prod_{s} \mathrm{~d} \psi_{s}}{\sqrt{\operatorname{det} 2 \pi \beta \omega\left(\mathbf{r}, \mathbf{r}^{\prime}\right)}}$ and $\omega^{-1}\left(\mathbf{r}, \mathbf{r}^{\prime}\right)$ is the inverse operator that satisfies the condition $\omega^{-1}\left(\mathbf{r}, \mathbf{r}^{\prime}\right) \omega\left(\mathbf{r}^{\prime}, \mathbf{r}^{\prime \prime}\right)=\delta\left(\mathbf{r}-\mathbf{r}^{\prime \prime}\right)$. Thus, the interaction energy is represented by the Green function for this operator and $v^{2}= \pm 1$ in accordance with the sign of the interaction of the potential energy. The introduced field variable $\varphi(\mathbf{r})$ contains information similar to the original distribution function, i.e., complete information concerning probable spatial states of the system. In this way introduced field which describe behavior of the distribution of the interacting particles.

The statistical operator can be rewritten in the form given by 


$$
Q_{l}=\int D \Gamma \int D \psi \exp \left\{-\int\left(\beta(\mathbf{r}) \frac{p^{2}(\mathbf{r})}{2 m(\mathbf{r})}-\eta(\mathbf{r})-i \sqrt{\beta(\mathbf{r})} \psi(\mathbf{r})\right) n(\mathbf{r}) \mathrm{d} \mathbf{r}\right\} Q_{i n t}
$$

where $Q_{i n t}=\exp \left\{-\frac{1}{2} \int \beta(\mathbf{r}) U\left(\mathbf{r}, \mathbf{r}^{\prime}\right)^{-1} \psi(\mathbf{r}) \psi\left(\mathbf{r}^{\prime}\right) \mathrm{d} \mathbf{r d} \mathbf{r}^{\prime}\right\}$ follows from the interaction in terms of the field variable. The latter general functional integral can be integrated over the phase space. We substitute the definition expression for the density, then the nonequilibrium statistical operator reduces to

$$
Q_{l}=\int D \psi \int \frac{1}{(2 \pi \hbar)^{3} N !} \prod_{i} \mathrm{~d} r_{i} \mathrm{~d} p_{i} \xi\left(\mathbf{r}_{i}\right) \exp \left\{-\left(\beta\left(\mathbf{r}_{i}\right) \frac{p_{i}^{2}}{2 m_{i}}-i \sqrt{\beta\left(\mathbf{r}_{i}\right)} \psi\left(\mathbf{r}_{i}\right)\right)\right\} Q_{i n t}
$$

where $\xi(\mathbf{r}) \equiv \exp \eta(\mathbf{r})$ is the chemical activity. Integration over all the moments reduces the real part of the non-equilibrium statistical operator to a simple expression [4] [9] given by

$$
Q_{l}=\int D \psi Q_{i n t} \exp \left\{\int\left[\xi(\mathbf{r})\left(\frac{2 \pi m(\mathbf{r})}{\hbar^{3} \beta(\mathbf{r})}\right)^{\frac{3}{2}} \cos (\sqrt{\beta(\mathbf{r})} \psi(\mathbf{r}))\right] \mathrm{d} \mathbf{r}\right\}
$$

For the general case of long-range interaction, e.g., Coulomb like or Newtonian gravitation interaction in continuum, the limiting inverse operator should be treated in the operator sense, i.e., $U^{-1}\left(\mathbf{r}, \mathbf{r}^{\prime}\right)=-L_{\mathbf{r r}^{\prime}}=-L_{\mathbf{r}^{\prime}} \delta\left(\mathbf{r}-\mathbf{r}^{\prime}\right)$. For the case of long-range interaction between particles, the non-equilibrium statistical operator can be written as

$$
Q_{l}=\int D \psi \exp \left\{\int\left[\psi(\mathbf{r}) L_{\mathbf{r r}^{\prime}} \psi\left(\mathbf{r}^{\prime}\right)+\xi(\mathbf{r}) \Lambda^{-3}(\mathbf{r}) \cos (\sqrt{\beta(\mathbf{r})} \psi(\mathbf{r}))\right] \mathrm{d} \mathbf{r}\right\}
$$

where $\Lambda(\mathbf{r})=\left(\frac{\hbar^{2} \beta(\mathbf{r})}{2 m(\mathbf{r})}\right)^{\frac{1}{2}}$ is the thermal de-Broglie wavelength in each spatial point. In the general case, the non-equilibrium statistical operator is given by

$$
Q_{l}=\int D \psi \exp \{S(\psi(\mathbf{r}), \xi(\mathbf{r}), \beta(\mathbf{r}))\}
$$

with the effective non-equilibrium "local entropy" being described by the expression

$$
S=\int\left[\psi(\mathbf{r}) L_{\mathbf{r r}} \psi\left(\mathbf{r}^{\prime}\right)+\xi(\mathbf{r}) \Lambda^{-3}(\mathbf{r}) \cos (\sqrt{\beta(\mathbf{r})} \psi(\mathbf{r}))\right] \mathrm{d} \mathbf{r}
$$

The statistical operator is suitable to apply the efficient methods developed in the quantum field theory without additional restrictions on either integration over the field variables or the perturbation theory. The functional $S(\psi(\mathbf{r}), \xi(\mathbf{r}), \beta(r))$ depends on the field distribution, the chemical activity, and the inverse temperature. After that we can apply the saddle-point method to find the asymptotic value of the statistical operator $Q_{l}$ as the number of particles $N$ tends to infinity. The dominant contribution is given by the states that satisfy the extremum condition for the functional. It can be easily shown that the saddle-point equation represents the thermodynamic relation and can be 
reduced to an equation for the field variable, $\frac{\delta S}{\delta \psi(\mathbf{r})}=0$, the normalization condition $\int \frac{\delta S}{\delta \xi(\mathbf{r})} \xi(\mathbf{r}) \mathrm{d} \mathbf{r}=N$, and the energy conservation law for the system $\int \frac{\delta S}{\delta \beta(\mathbf{r})} \xi(\mathbf{r}) \mathrm{d} \mathbf{r}=E$. The solution of this equation completely determines all the thermodynamical functions and describes the general behavior of interacting systems with both spatially homogeneous and inhomogeneous particle distributions. The above set of equations in principle solves the many-particle problem in the thermodynamical limiting case. The spatially inhomogeneous solution of these equations corresponds to the distribution of interacting particles. It is very important to note that only this approach makes it possible to take into account the inhomogeneous distribution of the temperature that can depend on the spatial distribution of particles in the system.

From the normalization condition and definition $\int \rho(\mathbf{r}) \mathrm{d} \mathbf{r}=N$ can introduce some new variable which present the macroscopic density function $\rho(\mathbf{r}) \equiv \Lambda^{-3}(\mathbf{r}) \xi(\mathbf{r}) \cos (\sqrt{\beta(\mathbf{r})} \psi(\mathbf{r}))$. In the case without interaction $\varphi(\mathbf{r})=0$ for free particles, if write the chemical activity in terms of the chemical potential $\xi(\mathbf{r})=\exp (\mu(\mathbf{r}) \beta(\mathbf{r}))$ from present definition can obtain the well-known relation $\beta(\mathbf{r}) \mu(\mathbf{r})=\ln \rho(\mathbf{r}) \Lambda^{3}(\mathbf{r})$ that generalizes the relation of the equilibrium statistical mechanics [17] [18]. The equation for energy conservation in this case can present in the new form:

$$
\frac{1}{2} \int \frac{\rho(\mathbf{r})}{\beta(\mathbf{r})}(3-\sqrt{\beta(r)} \psi(\mathbf{r}) \operatorname{tg}(\sqrt{\beta(\mathbf{r})} \psi(\mathbf{r}))) \mathrm{d} \mathbf{r}=E
$$

Derivation of the energy-conservation equation over the volume yields a relation for the chemical potential, i.e.,

$$
\frac{1}{2} \frac{\rho(\mathbf{r})}{\beta(\mathbf{r})}(3-\sqrt{\beta(r)} \psi(\mathbf{r}) \operatorname{tg}(\sqrt{\beta(\mathbf{r})} \psi(\mathbf{r})))=\frac{\delta E}{\delta V} \frac{\delta V}{\delta N}=\mu(\mathbf{r}) \rho(\mathbf{r})
$$

hence the chemical potential is given by

$$
\mu(\mathbf{r}) \beta(\mathbf{r})=\frac{3}{2}-\frac{1}{2} \sqrt{\beta(r)} \psi(\mathbf{r}) \operatorname{tg}(\sqrt{\beta(\mathbf{r})} \psi(\mathbf{r})) .
$$

This approach also provides the equation of state for the system within the context of the thermodynamic relation for pressure $P=\frac{1}{\beta} \frac{\delta S}{\delta V}$ for the case of energy conservation. The local equation of state is now reduced to

$$
P(\mathbf{r}) \beta(\mathbf{r})=\rho(\mathbf{r})\left(\mu(\mathbf{r}) \beta(\mathbf{r})-\frac{1}{2}\right)
$$

In the case of an ideal gas $\psi(\mathbf{r})=0$ we have $\mu \beta=\frac{3}{2}$ and obtain the usual equation of state $P \beta=\rho$. Thus the equation of state reproduces the equation of state of the ideal gas. The energy of the system is equal to $E=\frac{3}{2} N k T$, this 
formula is in accordance with the previous well-known results [18]. Within the context of the definition (15) we can conclude that, under the condition $\mu(\mathbf{r}) \beta(\mathbf{r})<\frac{1}{2}$, there appears negative pressure $P(\mathbf{r})<0$ that satisfies the necessary vacuum condition in the cosmology. It holds under the special condition $\sqrt{\beta(r)} \psi(\mathbf{r}) \operatorname{tg}(\sqrt{\beta(\mathbf{r})} \psi(\mathbf{r}))<2$, for constant temperature and for the total energy of the system $E<\frac{1}{2} N k T$. This condition implies that the energy of each particle is lower than the thermal energy. In this special case the energy of the system is lower than the total thermal energy of particles, that is impossible.

The possibility to apply the saddle-point method and thus to select the system states whose contributions in the partition function are dominant [6]. The solutions obtained equations associated with the finite values of the functional may be regarded as thermodynamically stable particle and field distributions. On this solution we must determine the general relation between thermodynamic parameter and their spatial dependence. Thus the spatially inhomogeneous distribution of the fields can be unambiguously related to the spatially inhomogeneous particle distribution. In general approach all thermodynamic parameter i.e. (pressure, chemical potential, density) dependence from spatial point and dependence one from other. Actually, this approach extends the average field approximation to involve into consideration spatially inhomogeneous field distributions. In our case the introduced field describes the nature of repulsive interaction and can present the fundamental scalar field which correspond for the scattering in the system.

If introduce the new field variable $\varphi=\sqrt{\beta(r)} \psi(\mathbf{r})$ and take into account the definition of the chemical potential (14) can rewrite the macroscopic density in the form given by

$$
\rho(\mathbf{r}) \equiv \Lambda_{e}^{-3}(\mathbf{r}) \exp \left\{-\frac{1}{2} \varphi(\mathbf{r}) \operatorname{tg} \varphi(\mathbf{r})\right\} \cos \varphi(\mathbf{r})
$$

where the de-Broglie wavelength is renormalized, i.e., $\Lambda_{e}=\left(\frac{\hbar^{2} \beta(\mathbf{r}) e}{2 m(\mathbf{r})}\right)^{\frac{1}{2}}$. The local entropy in terms of the new variable after substitution obtained relation in (11) is given by

$$
S=\int\left[\frac{\varphi(\mathbf{r})}{\sqrt{\beta(r)}} L_{\mathbf{r r}^{\prime}} \frac{\varphi\left(\mathbf{r}^{\prime}\right)}{\sqrt{\beta(r)}}+\Lambda_{e}^{-3}(\mathbf{r}) \exp \left\{-\frac{1}{2} \varphi(\mathbf{r}) \operatorname{tg} \varphi(\mathbf{r})\right\} \cos \varphi(\mathbf{r})\right] \mathrm{d} \mathbf{r}
$$

For constant temperature and equal particles masses the local entropy in the mean-field approximation can be presented as

$$
S=\int\left[\frac{1}{\beta} \varphi(\mathbf{r}) L_{\mathbf{r r}} \varphi\left(\mathbf{r}^{\prime}\right)+\Lambda_{e}^{-3} \exp \left\{-\frac{1}{2} \varphi(\mathbf{r}) \operatorname{tg} \varphi(\mathbf{r})\right\} \cos \varphi(\mathbf{r})\right] \mathrm{d} \mathbf{r}
$$


Now the equation for the field variable can be rewritten as

$$
2 L_{\mathbf{r}} \varphi\left(\mathbf{r}^{\prime}\right)-\beta \frac{\mathrm{d} V(\varphi)}{\mathrm{d} \varphi}=0
$$

where the potential energy $V(\varphi) \equiv \rho(\mathbf{r})=\Lambda_{e}^{-3} \exp \left(-\frac{1}{2} \varphi(\mathbf{r}) \operatorname{tg} \varphi(\mathbf{r})\right) \cos \varphi(\mathbf{r})$ is a function of the field variable in the above form. This potential has a minimum for $3 \sin 2 \varphi=-2 \varphi$. For small values of $\varphi$ we have two different solutions $\varphi=0$ and $\varphi^{2}=1$. For small $\varphi$ the effective potential is given by a very simple expression $V(\varphi)=\left(1-\varphi^{2}\right)$ and the equation for the field variable reduces to $2 L_{\mathrm{rr}} \varphi\left(\mathbf{r}^{\prime}\right)+2 \beta \varphi\left(\mathbf{r}^{\prime}\right)=0$. In the general case the potential energy of the field possesses oscillation character with decreasing amplitude. This fully relation with necessary condition of the cosmological model natural inflation was proposed in article [19]. After that we can analyze the probable spatial solution for the field variable and the behavior of the field in the time. In order to provide this, the knowledge of particle interaction energy with relevant specifics is required. It is well-known from cosmology reasoning that galaxy scattering is associated with the fundamental scalar field. We have shown above that the repulsive statistical interaction can be described in terms of the field $\varphi=\sqrt{\beta(r)} \psi(\mathbf{r})$. We suppose that the introduced fields are fundamental scalar fields responsible for the statistical motivation of the scattering of matter. From this assumption we can find the energy of interaction between two masses located in different spatial point. As follows from cosmology, two masses scatter with the velocity $v=H\left(\mathbf{r}-\mathbf{r}^{\prime}\right)$ where $H$ is the Hubble constant and $\mathbf{r}-\mathbf{r}^{\prime}$ is the distance between them. The kinetic energy of the relative motion for each mass is given by $T=\frac{m(\mathbf{r})}{2} H^{2}\left(\mathbf{r}-\mathbf{r}^{\prime}\right)^{2}$ and thus the energy of interaction between two masses located in different spatial points is $W\left(\mathbf{r}-\mathbf{r}^{\prime}\right)=\frac{m(\mathbf{r})}{2} H^{2}\left(\mathbf{r}-\mathbf{r}^{\prime}\right)^{2}+\frac{m\left(\mathbf{r}^{\prime}\right)}{2} H^{2}\left(\mathbf{r}-\mathbf{r}^{\prime}\right)^{2}$. For homogeneous distribution of masses, the last expression can be rewritten as $W\left(\mathbf{r}-\mathbf{r}^{\prime}\right)=m(\mathbf{r}) H^{2}\left(\mathbf{r}-\mathbf{r}^{\prime}\right)^{2}$. In terms of such interaction energy, the inverse operator is given by

$$
L_{\mathbf{r r}^{\prime}}=\frac{1}{m H^{2}} \frac{\mathrm{d}^{2}}{\mathrm{~d} r^{2}}
$$

Having determined the inverse operator, we can present the spatial dependence of the fundamental scalar field as the solution of the equation

$$
\frac{2}{m H^{2}} \frac{\mathrm{d}^{2} \varphi}{\mathrm{d} r^{2}}-\beta \frac{\mathrm{d} V(\varphi)}{\mathrm{d} \varphi}=0 .
$$

For small values of $\varphi$ the latter transforms to the equation

$$
\frac{\mathrm{d}^{2} \varphi}{\mathrm{d} r^{2}}+\beta m H^{2} \varphi=0
$$

that has a periodical solution $\varphi=\cos (\sqrt{m \beta} H r)$ with the spatial period $\frac{1}{\sqrt{m \beta} H}$. For distances shorter than this value we can regard the fundamental 
scalar field to be invariable. However, the fundamental scalar field can change in time. To describe the evolution of such fields, we can present a dynamical equation.

In our case, however, this field motivates the repulsive interaction in the system and the entropy increase. The behavior of the solution of the equation is similar to the behavior that follows from the usual equation for the scalar field and formation of a new-phase bubble of the fundamental scalar field. The present solution can describe the formation of a bubble of a new phase in the theory of inflation of the Universe [20] [21] [22], and the field variable introduced plays the role of the fundamental scalar field and takes into account the repulsive interaction in the system under consideration. In the general presentation, formula (11) can describe the condition of new phase formation, the size of the bubble, and other parameters of the thermodynamical behavior of such systems. This non-equilibrium statistical description concerns only probable dilute structures of such systems, it does not describe metastable states and tells nothing about the time scales in the dynamical theory. In this way, however, we can solve complicated problems of the statistical description of interacting systems. For this purpose we have to derive a dynamical equation for the field. In this sense we can use the Ginsburg-Landau equation for the fundamental scalar field in the standard form given by

$$
\frac{\partial \varphi(\mathbf{r}, t)}{\partial t}=-\gamma \frac{\delta S}{\delta \varphi(\mathbf{r})}
$$

where $\gamma$ is the dynamical viscosity coefficient [23]. In this case all the necessary conditions satisfy the thermodynamic relation. We can suppose that the motivation of the Universe dynamics is associated with the entropy increase. The evolution in the non-equilibrium state is governed by the local entropy landscape and the morphological instabilities of the parameter. The dynamics of the system is dissipative, and it should result in the decrease of the local entropy. This solution obtained equation which gives the answer on the equation: what is motive scattering of the matter. Dynamic of formed Universe can be only dissipative and for description of existence of Universe we must take into account its nonequilibrium conditions.

Interacting particle systems are non-equilibrium a priory. Before relaxing to the thermodynamic equilibrium, isolated systems with long-range interactions are trapped in non-equilibrium quasi-stationary states whose lifetimes diverge as the number of particles increases. A theory was presented which allows us to quantitatively predict the instability threshold for spontaneous symmetry breaking for a class of d-dimensional systems [24]. Non-equilibrium stationary states of the systems were described in article [25] which concluded that three-dimensional systems do not evolve to thermodynamic equilibrium but are trapped in non-equilibrium quasi-stationary states. We propose an approach that provides a possibility to quantitatively predict the particle distribution in a system with special repulsive interaction. In this way we can solve the compli- 
cated problem of the statistical description of systems with special repulsive interactions and introduce a new field variable that reduces this task to the solution of the cosmological problem. Moreover, this method may also be applied for the further development of physics of self-gravitating and similar systems that are not far from equilibrium.

\section{Acknowledgements}

This article carry out for financial support from theme department of physics and astronomy of NAS Ukraine "Dynamic formation spatial uniform structures in many-body system" PK 0118U003535. This work was supported in part by Brain Pool program by Grant N 218H1D3A2065894 through the National Research Foundation of Korea (NRF).

\section{Conflicts of Interest}

The author declares no conflicts of interest regarding the publication of this paper.

\section{References}

[1] Thirring, W. (1970) Zeitschrift für Physik, 235, 339-352. https://doi.org/10.1007/BF01403177

[2] Chavanis, P.-H., Rosier, C. and Sire, C. (2002) Physical Review E, 66, Article ID: 036105.

[3] Laliena, V. (2003) Nuclear Physics B, 668, 403-411. https://doi.org/10.1016/j.nuclphysb.2003.07.005

[4] Lev, B.I. and Zhugaevych, A.Ya. (1998) Physical Review E, 57, 6460. https://doi.org/10.1103/PhysRevE.57.6460

[5] Lev, B.I. and Zagorodny, A.G. (2011) Physical Review E, 84, Article ID: 061115. https://doi.org/10.1103/PhysRevE.84.061115

[6] Lev, B.I. (2011) International Journal of Modern Physics B, 25, 2237-2249. https://doi.org/10.1142/S0217979211100771

[7] Zubarev, D.N. (1974) Non-Equilibrium Statistical Thermodynamics. Consultans Bareu, New York.

[8] Kleinert, H. (1989) Gauge Fields in Condensed Matter. World Scientific, Singapore. https://doi.org/10.1142/0356

[9] deVega, H.J., Sanchez, N. and Combes, F. (1996) Physical Review D, 54, 6008. https://doi.org/10.1103/PhysRevD.54.6008

[10] Lipatov, L.N. (1977) JETP(Sov), 72, 412.

[11] Edward, S. and Lenard, A. (1962) Journal of Mathematical Physics, 3, 778. https://doi.org/10.1063/1.1724281

[12] Hubbard, J. (1959) Physical Review Letters, 3, 77. https://doi.org/10.1103/PhysRevLett.3.77

[13] Grigorishin, K.V. and Lev, B.I. (2005) Physical Review E, 71, Article ID: 066105. https://doi.org/10.1103/PhysRevE.71.066105

[14] Bilotsky, Y.D. and Lev, B.I. (1984) Teoreticheskaya i Matematicheskaya Fizika, 60, 120. 
[15] Samuel, S. (1978) Physical Review D, 18, 1916. https://doi.org/10.1103/PhysRevD.18.1916

[16] Stratonovich, R.L. (1958) Soviet Physics, Doklady, 2, 416.

[17] Ruelle, D. (1969) Statistical Mechanics. Rigorous Results, New York, Amsterdam.

[18] Huang, D. (1969) Statistical Mechanics. W. A. Benjamin, New York.

[19] Adams, F.C., Richard Bond, J., Freese, K., Frieman, J.A. and Olinto, A.V. (1993) Physical Review D, 47, 426. https://doi.org/10.1103/PhysRevD.47.426

[20] Linde, A.D. (1990) Elementary Particle Physics and Inflationary Cosmology. Horwood Academic, Chan. https://doi.org/10.1201/b16971

[21] Linde, A.D. (1979) Reports on Progress in Physics, 42, 389-437. https://doi.org/10.1088/0034-4885/42/3/001

[22] Coleman, S. (1977) Physical Review D, 15, 2929-2936. https://doi.org/10.1103/PhysRevD.15.2929

[23] Landau, L.D. (1973) Statistical Mechanics. Nauka, Moskwa.

[24] Pakter, R., Marcos, B. and Levin, Y. (2013) Physical Review Letters, 111, Article ID: 230603. https://doi.org/10.1103/PhysRevLett.111.230603

[25] Benetti, F.P.C., Ribeiro-Teixeira, A.C., Pakter, R. and Levin, Y. (2014) Physical Review Letters, 113, Article ID: 100602.

https://doi.org/10.1103/PhysRevLett.113.100602 